\title{
BMJ Open Using primary care-based paper and telephone interventions to increase uptake of bowel scope screening in Yorkshire: a protocol of a randomised controlled trial
}

\author{
Lesley M McGregor, ${ }^{1}$ Robert S Kerrison, ${ }^{1}$ Trish Green, ${ }^{2}$ Una Macleod, ${ }^{2}$ \\ Mark Hughes, ${ }^{3}$ Monica Gibbins, ${ }^{4}$ Stephen Morris, ${ }^{5}$ Colin Rees, ${ }^{6}$ \\ Christian von Wagner ${ }^{1}$
}

To cite: McGregor LM, Kerrison RS, Green T, et al. Using primary care-based paper and telephone interventions to increase uptake of bowel scope screening in Yorkshire: a protocol of a randomised controlled trial. BMJ Open 2018;8:e024616. doi:10.1136/ bmjopen-2018-024616

- Prepublication history and additional material for this paper are available online. To view these files, please visit the journal online (http://dx.doi. org/10.1136/bmjopen-2018024616).

Received 7 June 2018 Revised 12 June 2018 Accepted 25 June 2018

Check for updates

(C) Author(s) (or their employer(s)) 2018. Re-use permitted under CC BY-NC. No commercial re-use. See rights and permissions. Published by BMJ.

For numbered affiliations see end of article.

Correspondence to Dr Lesley M McGregor; l.mcgregor@ucl.ac.uk

\section{ABSTRACT}

Introduction Evidence suggests bowel scope screening (BSS) can significantly reduce an individual's risk of developing colorectal cancer (CRC). BSS for 55 year olds was therefore introduced to the English Bowel Cancer Screening Programme (BCSP) in 2013. However, the benefits are only gained from test completion and uptake is low (43\%). Primary care involvement has consistently shown benefits to cancer screening uptake and so this study aims to build on this knowledge and evaluate general practitioner (GP) practice led interventions designed to increase BSS attendance.

Methods and analysis A three-arm randomised controlled trial will be conducted to evaluate three interventions: one intervention for prospective BSS invitees (primer letter with locally tailored leaflet sent by an individual's GP practice) and two interventions for those who do not attend their BSS appointment (a self-referral letter sent by an individual's GP practice and a patient navigation call made on behalf of an individual's GP practice). The trial will be set in Yorkshire. Individuals soon to receive their invitation to attend BSS at one of the Hull and East Yorkshire Bowel Cancer Screening centre sites, will be randomly assigned to one of three groups: control (usual care; no input from GP practice), Intervention group A (primer letter/leaflet and a self-referral letter), Intervention group B (primer letter/ leaflet and a patient navigation call). Attendance data will be obtained from the BCSP database (via National Health Service (NHS) Digital) 3 months after the last intervention. Regression analysis will compare uptake, and additional clinical outcomes, across the three groups. The analysis will be multivariate and adjust for several covariates including gender and area-level deprivation.

Ethics and dissemination NHS ethical approval has been obtained from London-Harrow Research Ethics Committee. The results will be submitted for publication in a peerreviewed journal and presented at conferences. Trial registration number ISRCTN16252122; Pre-results.

\section{INTRODUCTION}

Bowel cancer (colorectal cancer, CRC) is the second leading cause of cancer-related deaths
Strengths and limitations of this study

- This trial builds on previous work indicating a positive impact of similar interventions.

- This trial follows on from a Yorkshire-based cancer report that interventions delivered through primary care are most effective at increasing uptake.

- While each intervention is delivered through primary care, the study is designed to have minimal influence on the workload of each practice.

- The study is limited to one region in England potentially limiting the generalisability of our findings.

- Hull and East Yorkshire Bowel Cancer Screening centre currently only has two sites offering bowel scope screening, limiting the number of general practices that are eligible to take part in this study.

in the UK. ${ }^{1}$ However, screening opportunities to help reduce mortality from CRC via early detection or prevention are available.

Since 2006 the National Health Service (NHS) has invited all men and women, aged 60-69 (now increased to 74), to complete a home-based guaiac faecal occult blood test $(\mathrm{gFOBt})$ every 2 years with the aim of detecting CRC in its early stages, when it is considered more treatable. More recently (from 2013), the English Bowel Cancer Screening Programme (BCSP) has introduced bowel scope screening (BSS) as an additional test to help reduce an individual's risk of getting CRC.

Preceding the introduction of BSS, a UK trial of the test with a 10-year follow-up, found BSS to reduce CRC incidence in those who were screened by $33 \%$ and mortality by $43 \% .^{2}$ Evidence of BSS maintaining its protective value for 17 years has recently been published. ${ }^{3}$ 


\section{BSS and the invitation process}

BSS involves having a one-off flexible sigmoidoscopy to find and remove polyps within the lower part of the large bowel (rectum and sigmoid colon), before they can become cancerous. Individuals registered with a general practice in England, will become eligible for BSS at the age of 55 and 2 months. A preinvitation letter introducing a forthcoming appointment letter is first sent to eligible individuals along with an 11-page information booklet. The appointment letter is then sent 2 weeks later and includes a specified appointment date and time (set 6 weeks in advance), which individuals are asked to confirm by either a phone call or the return of a reply slip. If no confirmation is received by the screening centre, an appointment reminder letter is sent, followed 2 weeks later by a cancellation later if still no confirmation is received in that time period. If confirmation is received, a consent form and enema kit is sent out approximately 1-2 weeks before the appointment. For those who do not attend their confirmed appointment, a letter to confirm cancellation of the BSS appointment is sent. No more contact is made with the individual until they are eligible for a different bowel cancer screening test (ie, gFOBt) at age 60 (see figure 1). However, the individual can make a self-referral and initiate a new appointment process up until this point.

\section{Uptake of BSS}

Despite the benefits of BSS, uptake of the test is low $(43 \%) .{ }^{4}$ Similar to gFOBt, uptake of BSS is socially graded with those living in more deprived areas less likely to take part. ${ }^{4}$ In contrast to gFOBt participation, males are significantly more likely than females to attend ( $45 \%$ vs $42 \%) .^{4}$ Interventions to enhance uptake and minimise inequalities as BSS continues to be rolled out across England are warranted.

Yorkshire and the Humber was one of the last regions in England to begin the BSS roll-out. Hull is a particularly deprived area within the region and has been identified as having one of the highest numbers of preventable cancer deaths in England (117.9 per 100000 compared with the English average of 79.4 per 100000$).{ }^{6}$ A high uptake of BSS could play a vital role in reducing the number of preventable cancer deaths and, as such, Hull was selected as the principal study setting.

\section{Developed interventions}

The interventions designed for this study have evolved from previous literature and are to be delivered external to the current programme structure. In a review of cancer screening participation interventions, general practitioner (GP) endorsement was found to have a reliably positive influence on uptake. ${ }^{7}$ However, the level of endorsement needed for the most positive effect is unclear. Within CRC screening, various degrees of positive outcomes have been documented using personalised letters signed by an individual's $\mathrm{GP},{ }^{8}$ and a simple statement to acknowledge GP practice support of the screening programme in another (ASCEND).$^{5}$

Within this study, we have opted to enhance the role of primary care endorsement within the screening context and have designed a letter, to be sent by an individual's GP practice ahead of the NHS invitation, to introduce BSS to patients and prime them for the NHS BCSP invitation process. This letter additionally builds on the literature to suggest a role for prescreening interventions. ${ }^{79}$ A further letter sent to non-attenders to remind them of the benefits of the test and the option to self-refer will also be included in this study. This intervention additionally draws on the literature suggesting a positive impact of personalised reminders on uptake. ${ }^{7}$

A more generic GP endorsement is also included in a leaflet designed as a brief introduction to BSS, with local tailoring to further invoke personalisation. Such a leaflet has been found to enhance BSS participation, over and above the standard NHS BSS information booklet, when included with a self-referral reminder sent 12 and 24 months after the original invitation. ${ }^{10}$

A further intervention included in this study is a patient navigation $(\mathrm{PN})$ call to BSS non-attenders from the individual's GP practice. PN was founded within the USA and was introduced to help minority groups identify and overcome barriers to access appropriate cancer care, and thereby lower socioeconomic health inequalities. ${ }^{11}$ PN offers one-to-one support to individuals/ patients and has been applied to CRC screening in the USA with positive outcomes (eg, refs ${ }^{12-14}$ ). A recent study tested the feasibility of PN to increase BSS uptake in England. ${ }^{15}$ Unfortunately, the process of asking prospective invitees for their telephone numbers at the start of the screening episode did not prove to be viable and did not allow the predicted value of PN to be evaluated. ${ }^{16}$ This study has been designed to try and overcome this limitation by embedding PN within primary care where patient numbers are readily available and calls can be initiated by a familiar healthcare provider. Based on evidence demonstrating the value of $\mathrm{PN}$, and UK evidence to support the use of GP practice based telephone calls to promote bowel cancer screening (eg, ref ${ }^{17}$ ), we hypothesise that this intervention will have the most influence on BSS uptake.

\section{METHODS AND ANALYSIS Design}

A randomised controlled trial with three parallel arms will test the impact of three interventions on uptake of BSS: one intervention will be aimed at BSS invitees and two at BSS non-attenders. A randomisation ratio of 1:1:1 will be applied for two intervention groups to be compared against each other and a control group of usual care (ie, no planned input from primary care; standard NHS BCSP contact procedures only). This will allow the additive value of the interventions to be considered in the analysis. Each intervention group 


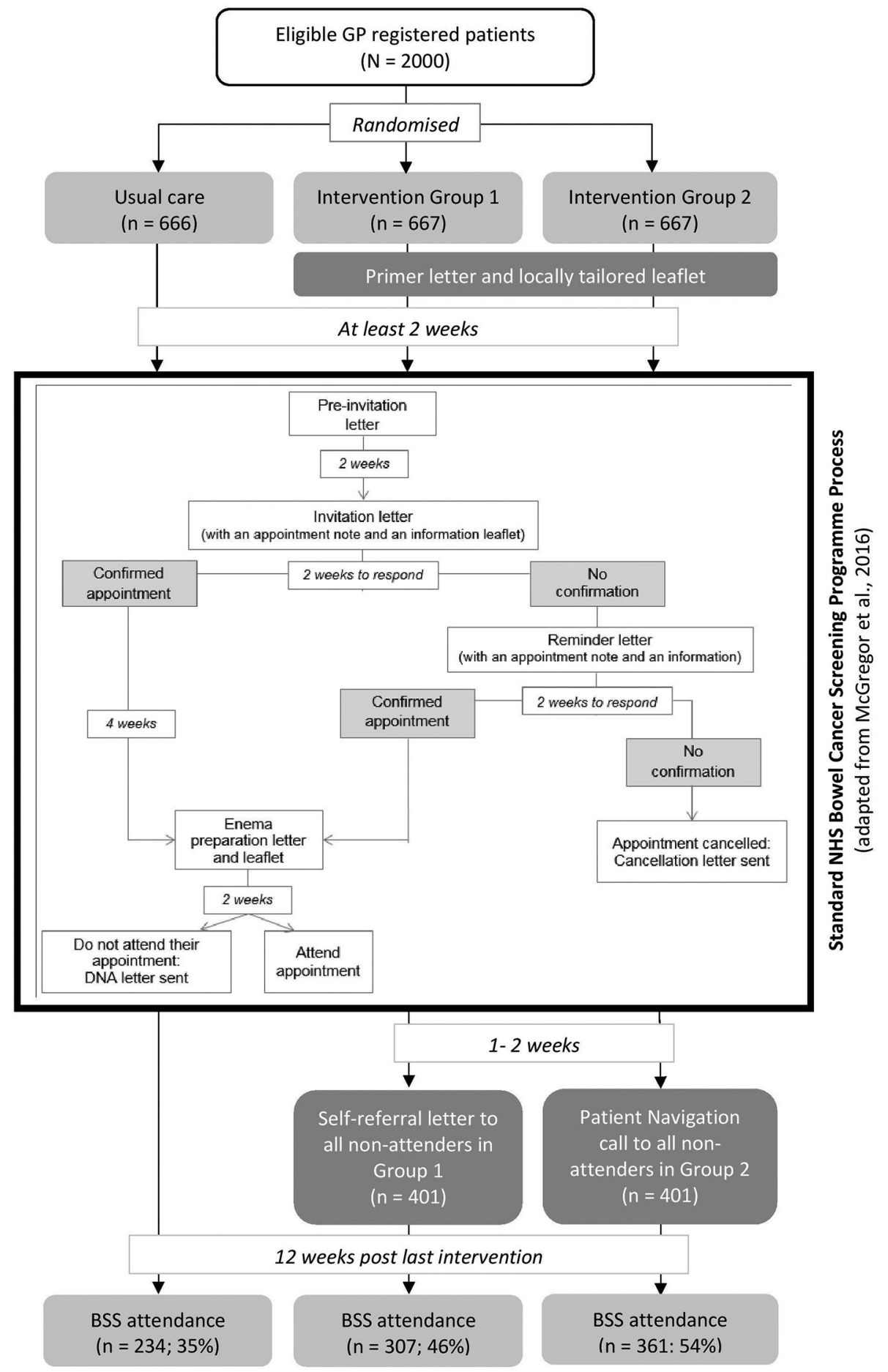

Figure 1 CONSORT flow diagram of process and expected attendance outcomes. Adopted from McGregor et al. ${ }^{15} \mathrm{BSS}$, bowel scope screening; CONSORT, Consolidated Standards of Reporting Trials; GP, general practitioner; NHS, National Health Service.

will include the invitee intervention and one of two non-attender interventions (see figure 1). At the end of data collection, an intervention process evaluation will be conducted involving telephone interviews with a selection of patients from each of the intervention groups, and a survey with staff at participating GP practices. The study is considered to be a low risk to patients and so will be 'centrally monitored' by the sponsor; any adverse events recognised by the GP practices will be reported to the sponsor.

\section{Recruitment and setting}

All general practices who are 'live' for BSS and linked to screening sites at Hull Royal Infirmary or Castle Hill Hospital (England, UK) will be invited to participate in this study; recruited practices will be listed on the trial 
register (ISRCTN16252122). As per the English NHS BCSP's structure, patients registered with a GP practice will become eligible for BSS at the age of 55 and 2 months. For this study, patients, who will become eligible for BSS in approximately 1-month time (ie, are currently aged 55 and 1 month) and fit the study inclusion criteria, will be automatically randomised into this study. No consent for study participation will be obtained from patients; a legal basis to allow access to confidential patient data without consent has been agreed (letter received 1 May 2018; CAG reference: 18/CAG/0049). However, each participating practice will display research notification posters to alert patients to this research project and will provide information on how to opt-out if they do not wish to be involved (see online supplementary appendices I and II for notification poster and leaflet).

\section{Inclusion criteria}

For an individual to be eligible for inclusion in the study, they must:

- Be registered with a participating GP practice.

- Be aged 55 years and 1 month (specifically, between 55 and 21 days and 55 years and 48 days) at the time they are enrolled in the study. By using this day-specific time frame for the monthly selection of people to be randomised into this study, we will ensure individuals assigned to one of the two intervention groups receive their primer letter at least 2 weeks before their formal NHS preinvitation letter.

\section{Exclusion criteria}

Individuals will not be eligible if:

- They do not meet the clinical eligibility criteria for BSS (ie, they have had their large bowel removed; they have a stoma bag to collect their stool; they are currently being treated for inflammatory bowel disease; they are awaiting heart surgery or have had heart surgery in the last 3 months, have heart or lung problems severely limiting physical abilities).

- They are registered on the general practice's clinical system as being a type 2 objector/opt-out.

- They are known to be receiving palliative care.

- They have been diagnosed with cancer (of any type) in the past 12 months.

Further exclusion criteria will be applied by each participating GP practice independently to ensure patients who would not normally be sent materials from the GP are not contacted. The reasons will be documented.

\section{Interventions}

The content of each intervention has been heavily influenced by local community work involving local residents and GP practice staff, and input from experts in public health, psychology, social marketing and health communication.

Primer letter and leaflet: A one-page letter priming patients to the delivery of the NHS invitation to attend BSS will be sent from their GP practice (GP practice headed paper and practice signatory) approximately 1 month before they are eligible for BSS. This letter will position the relevance of BSS for the individual by highlighting that their age is a risk factor for CRC and that the test can help prevent the second most common cause of cancer deaths. A threefold A5 leaflet entitled 'Bowel scope screening: a brief introduction' will be sent with the letter. The leaflet aims to engage patients with the topic of BSS without the pressure of a demand to make a decision about attendance, and content draws on theoretical models of behavioural change, for example, the Health Belief Model. ${ }^{18}$ The leaflet reiterates the seriousness of CRC and that age is a risk factor, irrespective of health status, provides key benefits and risks of the test, social norm information and quotes addressing known barriers (eg, anticipated pain) from BSS attendees, a GP recommendation for BSS and a visual representation of the protective function of BSS.

Self-referral reminder letter: A personally addressed, one A4 page, self-referral reminder letter will be sent to the patient from their GP practice. The letter will be sent in response to the GP practice receiving a notification from the screening hub indicating that their patient's appointment was cancelled (ie, they did not confirm or attend their BSS appointment). It will highlight the opportunity to self-refer up until the individual's 60 th birthday, but will recommend this is done sooner. In addition to providing the phone number to call to directly book a new appointment, a second A4 page will be a response slip to be completed and returned to the screening centre to request a call to arrange a new appointment. A Freepost return envelope will be provided. The slip will also encourage patients to consider and communicate appointment preferences and requirements that may have previously been a barrier to attendance, for example, expected sex of endoscopist or physical limitations, so that these can be addressed during the call.

PN phone call: A phone call to the patient will be made from a trained navigator on behalf of participating GP practices. As above, the call will be initiated in response to the GP practice receiving a notification of non-attendance from the screening hub. The call will aim to elicit the individual's barriers to BSS attendance, with suggestions and support to overcome them. Efforts will be made to correct misunderstandings about the purpose of BSS and what the test involves to facilitate informed choice. Support to arrange a new appointment will be offered. If questions or concerns arise during the PN call that the navigator cannot answer, a Specialist Screening Practitioner from the local screening centre will be consulted. The PN episode will begin approximately 1 week after the GP practice receives a copy of the hub cancellation letter and will include up to five call attempts on various days and times across a 2-week period. A letter from the GP practice will be sent to anyone who cannot be contacted to highlight to the patient that a call to discuss BSS was attempted and that they can self-refer if they still wish to attend. 


\section{Procedure}

A list of patients from each participating GP practice who will be age eligible for a BSS invitation in 1 month will be extracted from the GP clinical system on a monthly basis by a research nurse (RN). Patients meeting any of the exclusion criteria will be removed. The final list will be added to the practice-specific study database with an integrated pregenerated block randomisation list for study groups (provided by statistician). To minimise cross-over effects, the address of each patient entered into the database will be reviewed with those previously entered and individuals living at the same address will be assigned the same study group. The name, address and NHS number of each person assigned to one of the two intervention groups will be securely uploaded to an online mailing company by the $\mathrm{RN}$ working exclusively on this study. The mailing company will provide a secure process for the printing and delivery of all personalised letters. The 'return to sender address' will be the relevant GP practice and received returns will be noted on the study database.

The $\mathrm{RN}$ will then monitor the receipt of letters from the screening hub to inform the practice of their patients' attendance/cancellation, noting in the study database if patients randomised into the study have attended BSS or not. For those assigned to one of the intervention groups, who have not attended BSS, a self-referral intervention will be activated. Intervention $2 \mathrm{~A}$ will involve the RN uploading the name, address and NHS number of relevant patients to the online mailing company for the mail-out of the self-referral reminder letter. This will be sent approximately 1 week following receipt of the hub outcome letter. Intervention 2B will involve the RN finding relevant patients' telephone numbers from the GP clinical database and beginning the PN episode. The first call will be made 1 week after receipt of the hub letter. If no telephone contact is made after five attempts, a shortened version of the self-referral letter (eg no reply slip option included) will be sent via the online mailing company.

Three months following the last intervention, the RN will prepare two copies of each GP practice database. The first copy will include only the study ID and NHS numbers of all patients randomised into the study. This copy will be sent to NHS Digital who will use the NHS number to collate specified data from the bowel cancer screening system, for example, date of BSS attendance. Once collated, the data will be securely sent to the research team through University College London's (UCL) data safe haven with NHS numbers removed; data will not contain identifiable information. The second copy of the study database will be sent to the research team via UCL data safe haven with all personal details removed, for example, name, address, etc. The research team will combine the data received from each GP practice and NHS Digital using study ID numbers, in preparation for analysis (see figure 2).
Process evaluation

Where possible, the lead research team will randomly select patients to receive an invitation to be interviewed. Patients from each of the two intervention groups will be purposively selected: attended BSS following receipt of the primer letter and leaflet $(\mathrm{n}=33)$; attended following a self-referral intervention ( $\mathrm{n}=33$ ); did not attend following a self-referral intervention $(\mathrm{n}=33)$. Further invites will be sent depending on initial response rate with the aim of conducting an equal number of interviews across the outcome possibilities. The aim is to conduct 20 interviews (10 in each intervention group). The study ID numbers of selected patients will be sent to the $\mathrm{RN}$ who will obtain the corresponding names and addresses from the relevant GP practice database and upload them to the mailing company for the delivery of interview invitations (containing a GP-signed cover letter, information sheet, consent form and Freepost return envelope). Those interested will be asked to return the included consent form to the UCL researchers directly and a date and time for the interview will then be arranged. Participating patients will be offered a $£ 10$ voucher, which the interviewer will send to the address provided at the end of the interview.

The RN will oversee the delivery of an email with an embedded information sheet and link to the online survey to all staff in each of the participating GP practices. Confirmation of consent to use the data provided will be requested at the start of the survey. Staff members will be offered a chance to enter a draw to win a $£ 50$ voucher. Surveys will be completed anonymously; a separate link will direct participants to the prize draw whereby names and addresses can be entered separately to the online survey. All personal details received by the UCL research team will be securely held in the UCL Data Safe Haven until the payments are complete.

\section{Sample size}

The sample size $(n=2000)$ was calculated using a standard test of difference for two proportions. As the study contains three trial groups, the calculation was repeated for each pairwise comparison covering a primary research question in the planned analysis. The calculation giving the largest sample size requirement to test for a difference between any two of the three study groups was accepted and used as the sample size requirement for all three trial arms. The study has been designed to test for at least an $8 \%$ point difference in uptake between any two of the three study groups, with expected uptake values of $35 \%$, $46 \%$ and $54 \%$ in the usual care, primer and self-referral letter, and primer and PN groups, respectively (40\% for primer letter and leaflet alone), at the two-sided 5\% alpha level with a $20 \%$ margin for type II error.

To ensure we meet our target sample size, we need to recruit at least 35 GP practices 'live' for BSS and linked to the two screening centre sites in Hull. Recruitment initiatives will include incentives for early responders, presentations or stalls at primary care events, newsletter advertisements, targeted email notifications, practice 


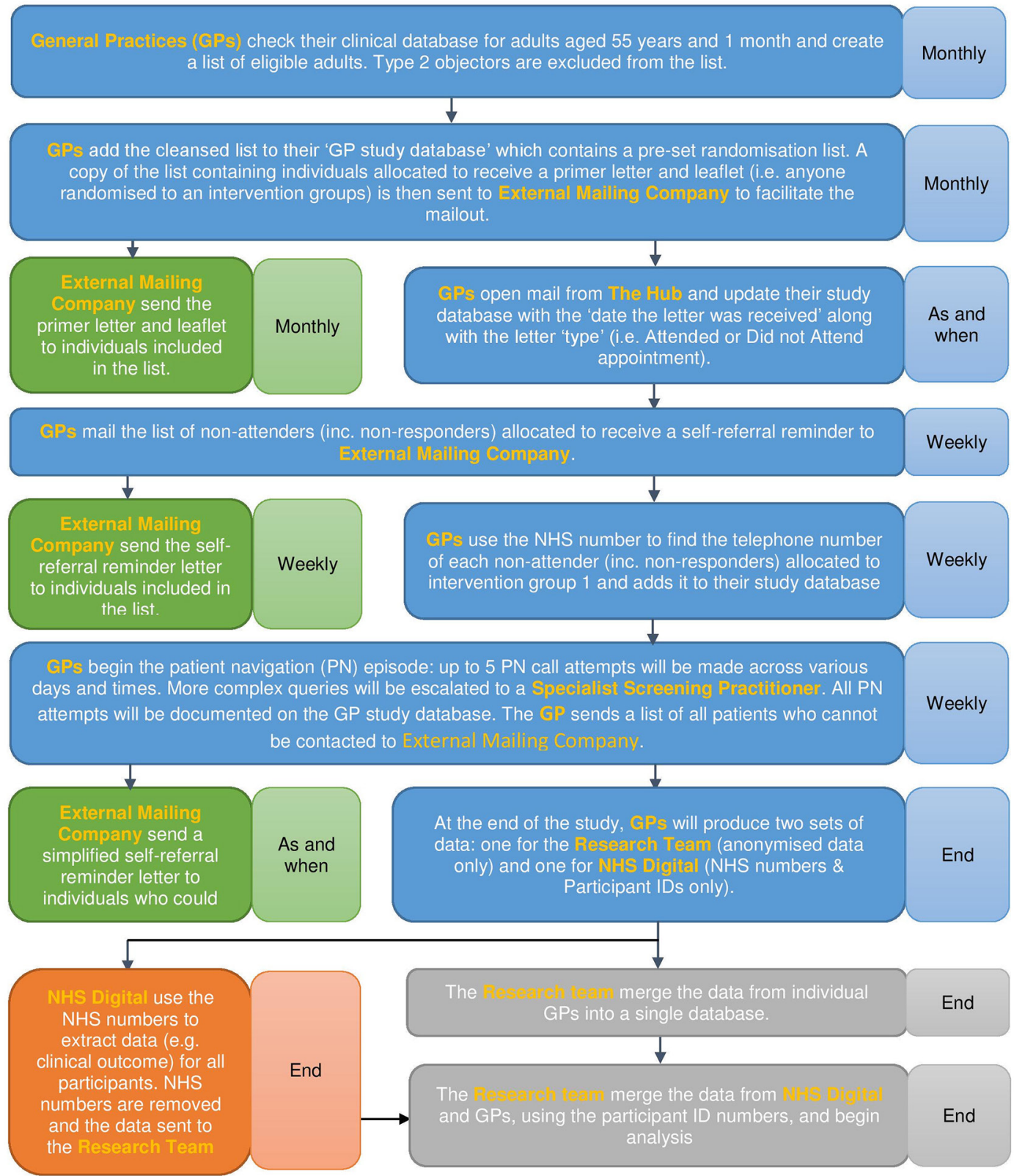

Figure 2 Study data flow diagram. NHS, National Health Service.

visits. The study is also supported by the National Institute for Health Research (NIHR) Clinical Research Network Yorkshire and the Humber.

\section{Outcomes measures}

The primary outcome measure will be the proportion of people who have attended BSS by 12 weeks post last intervention, within each group. Additional, secondary outcome measures will include, sociodemographic factors (eg, gender, Index of Multiple Deprivation (IMD) score, ethnicity) response to initial NHS invitation (did not respond or confirm/did respond or confirm); dates each intervention sent/calls made, date of attended BSS appointment, outcome of test (normal/abnormal/ cancer detected), offer of a colonoscopy (yes/no), attendance at colonoscopy (yes/no) and screening episode final clinical outcome (not suitable for BSS/normal/risk level/cancer).

\section{Planned analysis}

Univariable and multivariable binary logistic regression will be used to investigate the association between study groups and BSS attendance before and after adjusting for baseline characteristics (eg, sex, ethnicity, IMD quintile and response to the initial invitation). A subgroup analysis will consider whether the time interval between the primer letter and NHS invitation has an effect on uptake.

Further regression analyses will assess whether the interventions are predictors of attendance at colonoscopy for those offered a follow-up examination in response to their BSS appointment. The clinical outcomes for individuals randomised into the study will be compared with those 
who received usual care to consider the clinical impact of any increase in attendance found. SPSS statistical package (V.25.0+) will be used to manage the quantitative analysis.

A per-protocol analysis will test whether the difference between groups is stronger among those who were exposed to the intervention (as far as we can tell, eg, letters sent and not 'returned to sender', PN received vs attempted).

\section{Process evaluation}

Survey responses will be analysed descriptively. If the number of completed surveys allows, analysis of variance will be used to explore if views regarding the interventions and their impact differ between GP practices or staff groups (eg, GPs compared with healthcare assistants). A qualitative content analysis will be applied to any open-ended responses within the survey and to interview transcripts with patients. The analysis will be led by one researcher with a proportion second coded and discussed with members of the research team. Member checking of transcripts is not appropriate or necessary for the purposes of this evaluation. A data management tool (eg, NVivo or Quirkos) will be used to support analysis.

\section{Economic analysis}

At the end of the study, we will conduct an economic analysis to explore the potential of reducing treatment costs and increasing the quality-adjusted life years of the population through the reduced incidence and mortality of CRC. Pre-existing data on lifetime costs and benefits will be used in the analysis.

\section{Patient and public involvement}

This study benefits from having three patient and public representatives either in the core team (author MG) or in our advisory committee. All have been involved in the preparation of this study and have provided feedback on study design and/or intervention and notification materials. Public survey interviews to consider the proposed interventions were conducted initially, followed by codesign workshops with members of the local community to refine the content and format of the intervention materials. Our patient and public representatives will also have a role to play in the PN training process. As the study progresses our patient and public representatives will continue to be consulted and opportunities to be involved in dissemination of the results put forward.

\section{ETHICS AND DISSEMINATION}

All relevant approvals have been obtained (eg, Health Research Authority). The online mailing company has all the appropriate security measures in place (ie, ISO27001 certificate and NHS IG Toolkit) and a data processing agreement has been confirmed. The study protocol has been written in line with the Standard Protocol Items: Recommendations for Interventional Trials statement. ${ }^{1920}$ Any protocol amendments will be confirmed through the appropriate channels, the research team informed of the outcome and trial registration updated accordingly.

The results of the trial will be written up by the research team and published in peer-reviewed journals, presented at relevant conferences and reported on the website of the researchers and funders. A summary of the results will also be made available for each participating GP practice to disseminate to staff and patients.

\section{Author affiliations}

${ }^{1}$ Research Department of Behavioural Science and Health, University College London, London, UK

${ }^{2}$ Hull York Medical School, University of Hull, Hull, UK

${ }^{3}$ Hull and East Yorkshire Bowel Cancer Screening Centre, Hull and East Yorkshire Hospitals NHS Trust, Castle Hill Hospital, Hull, UK

${ }^{4}$ Patient Representative, Leicester, UK

${ }^{5}$ Department of Applied Health Research, University College London, London, UK ${ }^{6}$ South Tyneside NHS Foundation Trust, South Tyneside District Hospital, South Shields, UK

Acknowledgements Thanks are given to Nick Counsell for his guidance on the statistical design for this study and our other steering and advisory group committee members for their advice and contributions to the progress of this study so far. Further thanks are given to Graeme Duthie, former clinical director of the Hull and East Yorkshire Bowel Cancer Screening Centre, for his advice and guidance to the design of the study.

Contributors LMM and CvW are the principal investigators on this funded study and devised the research aims, objectives and methodology. LMM manages the approvals and recruitment for the trial, supervises RSK and drafted the initial manuscript, managed further iterations and submitted for publication. RSK is a coinvestigator on this study, contributed to the study design and has led the development of the intervention materials. UM is a coinvestigator on this study and contributed to the study design. TG is a coinvestigator on this study, contributed to the study design and has supported the development of study materials and General Practice recruitment. MH is a coinvestigator on this study, provides advice and guidance from the perspective of the local screening centre, and oversees the centre's role in this study. SM is a coinvestigator on this study and devised the cost analysis plans for the interventions included in this trial. CR is a coinvestigator on this study and contributed to the study design. MG is a coinvestigator on this study and offers advice and guidance on study design and documentation from the perspective of a patient and member of the public.

Funding This work is funded by Yorkshire Cancer Research (registered charity 516898; Award reference number UCL407).

Disclaimer Neither Yorkshire Cancer Research nor the study sponsors have had any input to the design of this study.

Competing interests None declared.

Patient consent Not required.

Ethics approval London-Harrow Research Ethics Committee.

Provenance and peer review Not commissioned; peer reviewed for ethical and funding approval prior to submission.

Open access This is an open access article distributed in accordance with the Creative Commons Attribution Non Commercial (CC BY-NC 4.0) license, which permits others to distribute, remix, adapt, build upon this work non-commercially, and license their derivative works on different terms, provided the original work is properly cited, appropriate credit is given, any changes made indicated, and the use is non-commercial. See: http://creativecommons.org/licenses/by-nc/4.0/.

\section{REFERENCES}

1. Cancer Research UK. Bowel Cancer Statistics. http://www. cancerresearchuk.org/health-professional/cancer-statistics/statisticsby-cancer-type/bowel-cancer (accessed 01 Jun 2018).

2. Atkin WS, Edwards R, Kralj-Hans I, et al. UK Flexible Sigmoidoscopy Trial Investigators. Once-only flexible sigmoidoscopy screening in prevention of colorectal cancer: a multicentre randomised controlled trial. Lancet 2010;375:1624-33. 
3. Atkin W, Wooldrage K, Parkin DM, et al. Long term effects of onceonly flexible sigmoidoscopy screening after 17 years of follow-up: the UK Flexible Sigmoidoscopy Screening randomised controlled trial. Lancet 2017:389:1299-311.

4. McGregor LM, Bonello B, Kerrison RS, et al. Uptake of Bowel Scope (Flexible Sigmoidoscopy) Screening in the English National Programme: the first 14 months. J Med Screen 2016;23:77-82.

5. Wardle J, von Wagner C, Kralj-Hans I, et al. Effects of evidencebased strategies to reduce the socioeconomic gradient of uptake in the English NHS Bowel Cancer Screening Programme (ASCEND): four cluster-randomised controlled trials. Lancet 2016;387:751-9.

6. Public Health England. Public health outcomes framework. https:// fingertips.phe.org.uk/profile/public-health-outcomes-framework/ data\#gid/1000044 (accessed 01 Jun 2018).

7. Duffy SW, Myles JP, Maroni R, et al. Rapid review of evaluation of interventions to improve participation in cancer screening services. $J$ Med Screen 2017;24:127-45.

8. Hewitson P, Ward AM, Heneghan C, et al. Primary care endorsement letter and a patient leaflet to improve participation in colorectal cancer screening: results of a factorial randomised trial. $\mathrm{Br} \mathrm{J}$ Cancer 2011;105:475-80.

9. Libby G, Bray J, Champion J, et al. Pre-notification increases uptake of colorectal cancer screening in all demographic groups: a randomized controlled trial. J Med Screen 2011;18:24-9.

10. Kerrison RS, McGregor LM, Counsell N, et al. Use of Two Selfreferral Reminders and a Theory-Based Leaflet to Increase the Uptake of Flexible Sigmoidoscopy in the English Bowel Scope Screening Program: Results From a Randomized Controlled Trial in London. Annals of Behavioral Medicine 2018;136:kax068.

11. Freeman HP. The origin, evolution, and principles of patient navigation. Cancer Epidemiol Biomarkers Prev 2012;21:1614-7.
12. Jandorf L, Gutierrez Y, Lopez J, et al. Use of a patient navigator to increase colorectal cancer screening in an urban neighborhood health clinic. J Urban Health 2005;82:216-24.

13. Escoffery C, Fernandez ME, Vernon SW, et al. Patient Navigation in a Colorectal Cancer Screening Program. J Public Health Manag Pract 2015;21:433-40.

14. DeGroff A, Schroy PC, Morrissey KG, et al. Patient Navigation for Colonoscopy Completion: Results of an RCT. Am J Prev Med 2017;53:363-72.

15. McGregor LM, Skrobanski H, Miller $\mathrm{H}$, et al. Using Specialist Screening Practitioners (SSPs) to increase uptake of the Bowel Scope (Flexible Sigmoidoscopy) Screening Programme: a study protocol for a feasibility single-stage phase II trial. Pilot Feasibility Stud 2016;2:54.

16. McGregor LM, Skrobanski H, Ritchie M, et al. von Wagner C. Using Specialist Screening Practitioners (SSPs) to increase uptake of Bowel Scope (Flexible Sigmoidoscopy) Screening: results of a feasibility single stage phase II randomised trial. Manuscript submitted.

17. Shankleman J, Massat NJ, Khagram L, et al. Evaluation of a service intervention to improve awareness and uptake of bowel cancer screening in ethnically-diverse areas. $\mathrm{Br} J$ Cancer 2014:111:1440-7.

18. Rosenstock IM, Strecher VJ, Becker MH. Social learning theory and the Health Belief Model. Health Educ Q 1988;15:175-83.

19. Chan A-W, Tetzlaff JM, Altman DG, et al. Statement: defining standard protocol items for clinical trials. Ann Intern Med 2013;2013:200-7.

20. Chan AW, Tetzlaff JM, Gøtzsche PC, et al. SPIRIT 2013 explanation and elaboration: guidance for protocols of clinical trials. BMJ 2013;346:e7586. 